\title{
BMJ Open Effects of implementing Pressure Ulcer Prevention Practice Guidelines (PUPPG) in the prevention of pressure ulcers among hospitalised elderly patients: a systematic review protocol
}

\author{
Amos Wung Buh, ${ }^{1}$ Hassan Mahmoud, ${ }^{2}$ Wenjun Chen (1) , 3,4 \\ Matthew D F Mclnnes, ${ }^{2,5,6}$ Dean A Fergusson (i) ${ }^{6}$
}

To cite: Wung Buh $\mathrm{A}$, Mahmoud $\mathrm{H}$, Chen W, et al. Effects of implementing Pressure Ulcer Prevention Practice Guidelines (PUPPG) in the prevention of pressure ulcers among hospitalised elderly patients: a systematic review protocol. BMJ Open 2021;11:e043042. doi:10.1136/ bmjopen-2020-043042

- Prepublication history and additional material for this paper is available online. To view these files, please visit the journal online (http://dx.doi.org/10. 1136/bmjopen-2020-043042).

AWB and HM contributed equally.

AWB and HM are joint first authors.

Received 23 November 2020 Revised 08 February 2021 Accepted 17 February 2021

Check for updates

(C) Author(s) (or their employer(s)) 2021. Re-use permitted under CC BY-NC. No commercial re-use. See rights and permissions. Published by BMJ.

For numbered affiliations see end of article.

Correspondence to

Wenjun Chen;

wchen140@uottawa.ca

\section{ABSTRACT}

Introduction Pressure ulcers are serious and potentially life-threatening problems across all age groups and across all medical specialties and care settings. The hospitalised elderly population is the most common group to develop pressure ulcers. This study aims to systematically review studies implementing pressure ulcer prevention strategies recommended in the Pressure Ulcer Prevention Practice Guidelines for the prevention of pressure ulcers among hospitalised elderly patients globally.

Methods and analysis A systematic review of all studies that have assessed the use of pressure ulcer prevention strategies in hospital settings among hospitalised elderly patients shall be conducted. A comprehensive search of all published articles in Medline Ovid, Cumulative Index to Nursing and Allied Health Literature, PubMed, Embase, Cochrane library, Scopus and Web of Science will be done using terms such as pressure ulcers, prevention strategies, elderly patients and hospital. Studies will be screened for eligibility through title, abstract and full text by two independent reviewers. Study quality and risk of bias will be assessed using the Joanna Briggs Institute for MetaAnalysis of Statistics Assessment and Review Instrument. If sufficient data are available, a meta-analysis will be conducted to synthesise the effect size reported as $O R$ with $95 \%$ Cls using both fixed and random effect models. $\mathrm{I}^{2}$ statistics and visual inspection of the forest plots will be used to assess heterogeneity and identify the potential sources of heterogeneity. Publication bias will be assessed by visual inspections of funnel plots and Egger's test.

Ethics and dissemination № formal ethical approval or consent is required as no primary data will be collected. We aim to publish the research findings in a peer-reviewed scientific journal to promote knowledge transfer, as well as in conferences, seminars, congresses or symposia in a traditional manner.

PROSPERO registration number CRD42019129088.

\section{BACKGROUND}

Pressure ulcers (PU) also known as pressure injuries are areas of localised damage to the skin and/or underlying structures due to

\section{Strengths and limitations of this study}

- This is a systematic review and meta-analysis of randomised controlled trials.

- This review will be the first to synthesise the evidence regarding the effectiveness of guidelines used in pressure ulcer prevention for elderly patients in hospitals and offer the highest level of evidence for informed decisions on use of Pressure Ulcer Prevention Practice Guidelines (PUPPG) in prevention pressure ulcers in the elderly patients in hospital.

- There may be heterogeneity of interventions used on eligible studies and incomplete information reported about the interventions in the literature which could limit our ability to statistically compare the effectiveness of interventions.

- The main limitation of this review might be scarcity of randomised controlled trials on the use of PUPPG for preventing pressure ulcers in elderly patients, publication bias and methodological quality of grey literature that shall be found.

pressure and/or friction and shear. ${ }^{1}$ They are serious and potentially life-threatening problems across all age groups from the very young to the very old and across all medical specialties and care settings. ${ }^{2}$ It has been documented that hospital admissions due to PU are $75 \%$ higher than admissions for any other medical conditions and that, the consequences of PU development in hospitalised patients are particularly serious. ${ }^{2}$ Patients with hospital admission PU are three times more likely to be discharged to long-term care facilities and mortality of these patients is twice that of patients without hospital admission PU. ${ }^{3}$ The cost of treatment of PU is 2.5 times than its prevention, and $\mathrm{PU}$ increases the length of stay in the hospital from 4 to 
30 days, decreases quality of life, and increases pain, morbidity and mortality. ${ }^{4}$

On international level, hospital-acquired PUs (sometimes called decubitus ulcers) are very common. ${ }^{5}$ Although many of these cases are preventable, their point prevalence in Canadian hospitals for example is measured to be $25.1 \%{ }^{6}$ Unfortunately, the high rates of such condition are associated with subsequent high burden on the healthcare system and the national economy considering the high cost of their management, and the frequent occurrence of associated significant morbidity and mortality. ${ }^{5}$ According to the Ontario Case Costing Initiative database in 2013 using the European Pressure Ulcer Advisory Panel (EPUAP) staging system, it was estimated that the cost of management of stage II ulcer is up to US\$40 000 and can reach more than double this price for managing a single case of stage IV ulcer. ${ }^{7}$ A good example of the burden that PU add to the national economy was measured in USA; it was estimated that hospital acquired PUs increase the financial expenses on healthcare systems between US\$6 and US $\$ 15$ billion annually. ${ }^{8}$

The National Pressure Ulcer Advisory Panel (NPUAP), the EPUAP and the Pan Pacific Pressure Injury Alliance (PPPIA $)^{9}$ have defined PU as a 'lesion or a trauma to the skin and/or underlying tissue usually over a bony prominence and is the result of undiminished pressure, or pressure combination with shear, friction and moisture'. It is a degenerative progress attributable to biological tissues (skin and underlying tissues) being exposed to pressure and shearing forces. The pressure constrains the proper blood circulation and causes cell death, tissue necrosis and the development of ulcers. ${ }^{9}$ While the quality of PU prevention and treatment has increased considerably over the past years, PUs remains a global concern because of its frequency of occurrence and negative consequences for patients and families as well as for the healthcare system. ${ }^{10}$ Incidence of PUs for hospitalised patients ranges from $9 \%$ to $18 \%$, among which the elderly population appears to be the most common group to develop the ulcers. ${ }^{11}$ At the same time, many elderly patients are more vulnerable to be 'stuck' at a certain stage of PU for a long period of time and sometimes for the remainder of their lives. ${ }^{12}$ This may result in longer length of hospital stay, heavier burdens for the healthcare system and family members, worst quality of life for elderly patients, which may also influence their mental health such as emotional stability. ${ }^{13} 14$

NPUAP, EPUAP and PPPIA ${ }^{9}$ developed the Pressure Ulcer Prevention Practice Guideline (PUPPG), which involves a range of evidence-based recommendations for PUs prevention that could be applied by healthcare professionals globally. Frequently used PU prevention strategies recommended in this guideline includes PU risk assessment, regular repositioning, prevention management plan, appropriate use of support surfaces and protection, continence management, patient education, skin protection, nutritional assessment and adequate nutrition. ${ }^{15}$ It also includes some recommendations specifically for elderly people-'protect aged skin from skin injury associated with pressure and shear forces', taking into consideration that an aged person's skin is vulnerable. ${ }^{15}$

A number of studies have been conducted on the implementation of PU prevention strategies among hospitalised patients. One cluster randomised trial conducted in Canada revealed that multidisciplinary PU prevention groups are more cost effective than usual care and yields no significant improvement in the treatment of PUs. ${ }^{16}$ Despite the existence of the guidelines on the prevention of $\mathrm{PU}$, their effective utilisation in preventing PUs among hospitalised elderly patients varies in settings and countries. Also, although a number of studies have assessed strategies used in preventing PUs, there appears to be little or no information on systematic reviews that have assessed the effectiveness of guidelines used in PU prevention for elderly patients in hospitals. This study, therefore, aims to systematically review studies implementing PU prevention strategies recommended in the PUPPG for the prevention of PUs among hospitalised elderly patients globally.

\section{OBJECTIVE}

The objective of this review is to assess the effectiveness of each of the strategies included in the PUPPG guideline in reducing the incidence and prevalence of hospital acquired PUs in hospitalised elderly patients in comparison to no strategy (usual practice), or other strategies. The review question is: what is the effectiveness of implementing each of the PU prevention strategies included in the PUPPG in decreasing the incidence and prevalence of PUs among hospitalised elderly patients compared with no strategies (basic usual care) or different prevention strategies?

\section{METHODS}

\section{Study design}

This will be a systematic review and meta-analysis of published and unpublished studies that have assessed the use of PU prevention strategies in hospital settings among hospitalised elderly patients. The systematic review protocol has been developed and reported following the Preferred Reporting Items for Systematic Reviews and Meta-Analyses (PRISMA) criteria (see online supplemental appendix 1$).{ }^{17}$

\section{Inclusion criteria}

\section{Population included}

This systematic review will focus on studies that involved all vitally stable (not admitted in the intensive care unit) bed ridden hospitalised patients aged 60 or above.

\section{Interventions}

All studies that assessed the effect of PU preventive strategies found in the PUPPG, that were implemented on vitally stable bed ridden hospitalised patients aged 60 and 
above with an aim to decrease the occurrence of PUs, will be included in this review. Interventions will be limited to use of risk assessment, skin assessment, skin care, nutrition, position and repositioning, education and training, and medical devices care.

\section{Comparator}

Interventions will be compared with other strategies to identify the most effective among them and/or will also be compared with no interventions (regular basic management).

\section{Outcomes}

In this study, the primary outcome will be directly related to the incidence of the disease among elderly hospitalised patients (incidence shall be considered as the proportion of hospitalised patients who developed PUs while in hospital). Included studies must measure study duration related incidence of the disease and/or its point prevalence and / or stage of PU (severity) as a measure of the effectiveness of the preventive strategies.

\section{Types of studies}

We will focus only on Quantitative studies-experimental and quasi-experimental studies. These might include randomised and non-randomised controlled trials in addition to comparative and before-and-after studies.

\section{Language}

Only studies written in English will be included in this systematic review.

\section{SEARCH STRATEGY}

We will use a three-step strategy to find published and unpublished studies on PUs and their management. First, we will conduct an initial search through the Medline Ovid database using an analysis of text words found in the title and abstract, and the index terms used to describe the article. Second, we will use identified keywords and index terms to search for studies in identified databases. Finally, we will use the reference list of selected studies from the first and second searches to look for additional studies not found in the databases. For this study, we will consider only studies either published or unpublished in English.

The databases that shall be searched for this review will include Medline Ovid, Cumulative Index to Nursing and Allied Health Literature, PubMed, Embase, Cochrane library, Scopus and Web of Science. See online supplemental appendix 2 for the example searching strategy and results in Medline (Ovid). All these databases will provide published studies. To find unpublished studies on our topic, we will use Google, Grey Literature reports and the Centers for Disease Control and Prevention.

The keywords we will use for our initial searches in Medline Ovid will include 'pressure ulcers', 'pressure sore', 'bed sore', 'pressure injuries', 'prevention strategies', 'elderly patients' and 'hospital'.

\section{Study screening and selection}

The titles, abstracts and full text of studies selected for this study will be reviewed by two independent researchers to identify studies that potentially meet the inclusion criteria outlined above. The Covidence software will be used for title, abstract and full-text screening. After importing references and inclusion/exclusion criteria into the Covidence software, two independent reviewers will screen titles of included studies according to the eligible criteria. Conflicts between those two reviewers will be resolved through discussion with a third reviewer. The same procedures shall be used for abstract screening. Following the abstract screening, full texts of these potentially eligible studies will be retrieved and independently assessed for eligibility by two reviewers. Any disagreement between the two reviewers over the eligibility of a particular study will also be resolved through discussion with the third reviewer. The process of study selection will be reported using the PRISMA flow diagram. ${ }^{17}$

\section{Assessment of methodological quality}

Two independent reviewers will be used to assess the methodological validity of the quantitative papers that will be selected for retrieval prior to their inclusion in the review using standard critical appraisal tools from the Joanna Briggs Institute for Meta-Analysis of Statistics Assessment and Review Instrument (see online supplemental appendix 3). All disagreement between the two reviewers shall be settled through discussions.

\section{Data extraction}

After screening and selecting studies, key information from those studies will be extracted into an excel sheet for further analysis. We shall use a data extraction tool adapted from the standardised data extraction tool from the Joanna Briggs Institute Meta-Analysis of Statistics Assessment and Review Instrument (JBI-MAStARI). Considering the information, we will need for the data synthesis of our study, we shall use the JBI-MAStARI to develop a data extraction tool specifically for quantitative research data extraction (see online supplemental appendix 4). The tool will be used to extract: (1) Study characteristics of reviewed papers, such as authors, year of publication, journal; (2) Methods of the study, including study design (randomised control trial (RCT), quasi-RCT, longitudinal, retrospective), research purpose and/or questions; (3) participant characteristics, country where the study took place, setting, population, sample size, age, sex, ethnicity, socioeconomic status and/ or education level; (4) PU prevention strategies used in experimental group and control group (if applicable), (5) outcome measures and results and (6) conclusions of reviewed papers and any comments from reviewers. Two reviewers will independently perform data extraction. Authors of reviewed papers will be contacted in case of any missing details about their studies.

\section{Data synthesis}

A meta-analysis of outcomes combining various studies included in the review shall be done. We will assess 
statistical heterogeneity with $\mathrm{I}^{2}$, which will indicate the percentage of the total variation across studies: $0 \%-40 \%$ low heterogeneity, $30 \%-60 \%$ moderate heterogeneity, $50 \%-90 \%$ may represent substantial heterogeneity and $75 \%-100 \%$ is considerable heterogeneity. If there is a substantial amount of heterogeneity $(75 \%)$, then sources of heterogeneity will be examined through subgroup and sensitivity analyses. We will also use $\chi^{2}$ test to test the heterogeneity and consider $\mathrm{p}<0.05$ as statistically significant. A fixed-effects model will be selected for significant homogeneous studies; otherwise we will apply a randomeffects model. All outcomes will be summarised using ORs and 95\% CI. An OR $<1$ will represent a lower rate of outcome among the group of patients who were treated following the guidelines. Publication bias will be assessed by visual inspections of funnel plots and Egger's test.

We will also provide a narrative synthesis of the findings from the included studies. The narrative synthesis shall be structured by describing the studies according to the type of intervention used. This will include the three categories of interventions recommend in the PUPPG guideline $^{9}$ :

1. Prevention of PUs, including risk factors and risk assessment, skin and tissue assessment, preventive skin care and emerging therapies for prevention of PUs.

2. Interventions for prevention and treatment of PUs, such as nutrition in PU prevention and treatment, repositioning and early mobilisation, repositioning to prevent and treat PUs, support surface and medical device-related PUs.

3. Treatment of PUs, for example, assessment of PUs and monitoring of healing, pain assessment and treatment, wound care, assessment and treatment of infection and biofilms, wound dressings for treatment of PUs and surgery for PUs. Results will be presented in tables, figures and graphs, followed by discussion.

Publication bias will be assessed in all analyses synthesising 10 or more studies to ensure adequate power in the analysis. ${ }^{18}$ For investigation of the effect of small studies and publication bias, data from included studies will be entered into a funnel plot asymmetry test if we have at least 10 studies in the meta-analysis. Egger's statistical test will be implemented using STATA/SE V.13 (StataCorp). The quality of supporting evidence will be assessed by the Grades of Recommendation, Assessment, Development and Evaluation. ${ }^{19}$

\section{Patient and public involvement}

No patient involved.

\section{Ethics and dissemination}

This review will only use published literature and will not recruit participants. Therefore, no formal ethical approval or consent is necessary. It is anticipated that this systematic review will provide a detailed summary of the evidence of the effectiveness of the PUPPG in preventing the occurrence of PUs among elderly patients in hospital. It is also expected that the study will provide recommendations on the best PU preventive strategies applicable in healthcare settings. We aim to publish the research findings in a peer-reviewed scientific journal to promote knowledge transfer, as well as in various media, such as: conferences, seminars, congresses or symposia in a traditional manner.

\section{Author affiliations}

${ }^{1}$ Interdisciplinary School of Health Sciences, University of Ottawa, Ottawa, Ontario, Canada

${ }^{2}$ School of Epidemiology and Public Health, Faculty of Medicine, University of

Ottawa, Ottawa, Ontario, Canada

${ }^{3}$ School of Nursing, University of Ottawa, Ottawa, Ontario, Canada

${ }^{4}$ Xiangya School of Nursing, Central South University, Changsha, Hunan, China

${ }^{5}$ Department of Radiology, University of Ottawa, Ottawa, Ontario, Canada

${ }^{6}$ Clinical Epidemiology Program, Ottawa Hospital Research Institute, Ottawa,

Ontario, Canada

Acknowledgements The authors would like to thank Lindsey Sikora (librarian) for counselling in developing the searching strategies.

Contributors AWB, HM and WC contributed to the conception of the research question and writing of the protocol. HM, AWB, WC, MDFM and DAF contributed to the development of search strategies, eligibility criteria and methodology for data synthesis. HM, AWB, WC, MDFM and DAF contributed to drafting of the protocol and provided approval for the final version of this protocol. HM, AWB and WC will work in duplicate to screen the titles and abstracts of all the materials obtained using the search strategy to exclude the articles that do not meet the eligibility criteria. HM, AWB and WC will evaluate the potentially eligible studies with the full text and further exclude studies with documentation of the reason for exclusion. All authors will contribute to the bias assessment strategy and data extraction criteria. HM, AWB and WC will independently extract data from the included studies. HM, AWB and WC will analyse the data and draft the manuscript. All authors will read, provide feedback and approve the final manuscript.

Funding This work was supported by Hunan Provincial Key Laboratory of Nursing, grant number (2017TP1004), Hunan Provincial Science and Technology Department, China.

Competing interests None declared.

Patient consent for publication Not required.

Provenance and peer review Not commissioned; externally peer reviewed.

Supplemental material This content has been supplied by the author(s). It has not been vetted by BMJ Publishing Group Limited (BMJ) and may not have been peer-reviewed. Any opinions or recommendations discussed are solely those of the author(s) and are not endorsed by BMJ. BMJ disclaims all liability and responsibility arising from any reliance placed on the content. Where the content includes any translated material, BMJ does not warrant the accuracy and reliability of the translations (including but not limited to local regulations, clinical guidelines, terminology, drug names and drug dosages), and is not responsible for any error and/or omissions arising from translation and adaptation or otherwise.

Open access This is an open access article distributed in accordance with the Creative Commons Attribution Non Commercial (CC BY-NC 4.0) license, which permits others to distribute, remix, adapt, build upon this work non-commercially, and license their derivative works on different terms, provided the original work is properly cited, appropriate credit is given, any changes made indicated, and the use is non-commercial. See: http://creativecommons.org/licenses/by-nc/4.0/.

\section{ORCID iDs}

Wenjun Chen http://orcid.org/0000-0001-5398-8508

Dean A Fergusson http://orcid.org/0000-0002-3389-2485

\section{REFERENCES}

1 Stadnyk B, Mordoch E, Martin D. Factors in facilitating an organisational culture to prevent pressure ulcers among older adults in health-care facilities. J Wound Care 2018;27:S4-10.

2 Nursing in Practice. Management of pressure ulcers | nursing in practice. Available: https://www.nursinginpractice.com/article/ management-pressure-ulcers [Accessed 30 Jan 2019]. 
3 Cano A, Anglade D, Stamp H, et al. Improving outcomes by implementing a pressure ulcer prevention program (PUPP): going beyond the basics. Healthcare 2015;3:574-85.

4 Dalvand S, Ebadi A, Gheshlagh RG, Ghanei Gheshlagh R. Nurses' knowledge on pressure injury prevention: a systematic review and meta-analysis based on the pressure ulcer knowledge assessment tool. Clin Cosmet Investig Dermatol 2018;11:613-20.

5 Sullivan N, Schoelles KM. Preventing in-facility pressure ulcers as a patient safety strategy: a systematic review. Ann Intern Med 2013;158:410-6.

6 Woodbury MG, Houghton PE. Prevalence of pressure ulcers in Canadian healthcare settings. Ostomy Wound Manage 2004;50:36-8.

7 Chan B, leraci L, Mitsakakis N, et al. Net costs of hospital-acquired and pre-admission pus among older people hospitalised in Ontario. $J$ Wound Care 2013;22:341-6.

8 Markova A, Mostow EN. Us skin disease assessment: ulcer and wound care. Dermatol Clin 2012;30:ix:107-11.

9 NPUAP, EPUAP, PPPIA. Prevention and Treatment of Pressure Ulcers: Quick Reference Guide [Internet], 2014. Available: https:// www.npuap.org/wp-content/uploads/2014/08/Updated-10-16-14Quick-Reference-Guide-DIGITAL-NPUAP-EPUAP-PPPIA-16Oct2014. pdf [Accessed $1 \mathrm{Mar}$ 2019].

10 Chaboyer W, Bucknall T, Gillespie B, et al. Adherence to evidencebased pressure injury prevention guidelines in routine clinical practice: a longitudinal study. Int Wound J 2017;14:1290-8.
11 Bredesen IM, Bjøro K, Gunningberg L, et al. The prevalence, prevention and multilevel variance of pressure ulcers in Norwegian hospitals: a cross-sectional study. Int J Nurs Stud 2015;52:149-56.

12 Garcia AD, Thomas DR. Assessment and management of chronic pressure ulcers in the elderly. Med Clin North Am 2006;90:925-44.

13 Chiari P, Forni C, Guberti M, et al. Predictive factors for pressure ulcers in an older adult population hospitalized for hip fractures: a prognostic cohort study. PLOS One 2017;12:e0169909.

14 WHO. Mental health of older adults [Internet], 2017. Available: https://www.who.int/news-room/fact-sheets/detail/mental-health-ofolder-adults [Accessed 01 Mar 2019].

15 Latimer S, Chaboyer W, Gillespie B. Pressure injury prevention strategies in acute medical inpatients: an observational study. Contemp Nurse 2016;52:326-40.

16 Stern A, Mitsakakis N, Paulden M, et al. Pressure ulcer multidisciplinary teams via telemedicine: a pragmatic cluster randomized stepped wedge trial in long term care. BMC Health Serv Res 2014;14:83.

17 BMJ. Preferred reporting items for systematic review and metaanalysis protocols (PRISMA-P) 2015: elaboration and explanation | The BMJ [Internet]. Available: https://www-bmj-com.proxy.bib. uottawa.ca/content/349/bmj.g7647 [Accessed 20 Jul 2020].

18 Cochrane. Cochrane Handbook for systematic reviews of interventions. Available: /handbook/current [Accessed 20 Jul 2020].

19 Guyatt GH, Oxman AD, Vist GE, et al. Grade: an emerging consensus on rating quality of evidence and strength of recommendations. BMJ 2008;336:924-6. 